Military Technical College Kobry El-Kobbah, Cairo, Egypt

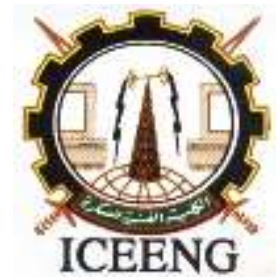

\title{
An Alternative Method to Simulate Three-Dimensional Point Scan Sensors aboard Moving Vehicle
}

\author{
M. Mosleh ${ }^{*}$ Gamal Abbas Zaghloul ${ }^{* *}$ Hany M. Arnaoot ${ }^{* * *}$
}

\begin{abstract}
This paper introduces an alternative simulation algorithm of a Laser Measurement System LMS sensor. The sensor is mounted on an autonomous ship sailing. The ship identifies the environment (surrounding fixed or moving obstacles) for safe navigation through the sensor. The simulation is done with six degree of freedom.

The proposed algorithm was applied by a vb.net 2010 program - written as part of the research- to do the simulation of the proposed model to reduce calculation overhead and to allow creation of embedded system.

The simulation algorithm was verified by in field sea trials on an $11 \mathrm{~m}$ long yacht with two 250 hp outboard engines. Sick 218-laser measurement system was used. The results showed that ship relatively high (roll, pitch) motion dynamics generates false data. It produces a large number of fake points (sea in case of unmanned surface vehicle or ground surface in case of robot moving on ground), which greatly overloads data processing system and in the same time reduces the target-detected points. This leads to reduction of the capability of detection .

This paper suggests a number of solutions to reduce this effect were suggested in this paper.

This paper is a step toward building an autonomous vehicle that can move in complex environment using a Point Scan Sensor as main sensor.
\end{abstract}

\section{KEY WORDS}

Point Scan Sensor, Simulation, Object Detection, Autonomous Navigation, LMS, Unmanned Surface Vehicle.

* Naval Architecture and Marine Engineer Dept., Faculty of Engineering, Alexandria University, Alexandria.

** Mechanical Power Engineering Dept., Faculty of Engineering, Port Said University.

${ }^{* * *}$ Egyptian Armed Forces. 


\section{Introduction}

Over the past few years, autonomous vehicle number and application increased rapidly. It is not limited to a specific field or application. Air Unmanned aerial vehicles (UAVs) have been widely used in various areas ranging from military to entertainment applications, because of reliable control and rapid movement. Recently, these vehicles have been used for searching dangerous or unexpected environments [1]. At sea there are several applications like autonomous underwater vehicles marine geosciences [2], autonomous unmanned merchant vessel [3]. Therefore, on-board automatic obstacle avoidance systems may be needed to safely support multiple vessel operations in cluttered complex transit environments [4. [

Operator visual monitoring and manual control may be inadequate in complex, cluttered transit environments. The success of the mobile robot navigation control depends mostly on the accuracy of absolute measurements of its position [5]. It is known that accurate navigation and surrounding data means accurate decision and vice versa.

Along with the increasing use of Laser Measurement Systems LMS, comes the need for a stable and accurate simulation system [6].Laser Measurement System is an examples of point scan sensors [7. [

Laser Measurement system sensors have been simulated using modern computer graphics hardware making heavy use of vertex and fragment shaders [8].

It worth mentioning that 3D calculation requires large computer resources and leads to delay [9].It is preferred to avoid using 3D calculation whenever it is possible. The suggested algorithm is meant to simulate point-scanning systems not line scanning systems [7].

\section{Research Gap}

Due to the fact that advance in applications require advance in design, and Research that involves the use of autonomous vehicle is generally divided into three phases:[10], [11]

1. Simulations

2. Navigation, guidance and control experiments

3. Create Application scenarios.

The current search is the first step of designing an autonomous ship by creating simulation of range sensors (such as laser, sonar, radar) which are used extensively in the field of robotics for constructing global terrain maps for object avoidance [12, 13]. The sensor data along with navigation data could be fed forward to any under test obstacle avoidance and navigation control. This simulation save time and money and provide a method for testing even the most complicated environment.

The developed algorithm could be used in the testing phase to validate control hardware efficiency. The test can be done through the use of a Hardware in the Loop (HIL) which is obtained by creating interface port that transmit simulated data and taking received autonomous control decision (in case of a ship the required rudder angle and required shaft speed) as inputs for the created HIL.

the proposed algorithm could be used when the sensor scan rate is high compared to its extrinsic motion (i.e. motion distortion within the scans can be neglected) or when the sensor scan rate is compared to its extrinsic motion [14]. 
The proposed algorithm in the current paper meant to predict Point Scan Sensor output data mounted on a moving vehicle (i.e. ground vehicle, ship, submerged under water vehicle......) detecting moving objects in different situation in three dimensions.

For the current case of Unmanned Surface Vehicle the sensor carrying ship as well as the objects dynamic data (X,Y, Roll and Yaw ) are calculated using Nomoto model[15].

The model presented by Nomoto does not include pitch angle estimation, so pitch angle is not included in calculations. However, current research methodology includes formula for the effect of pitch angle if available. The suggested algorithm has been deployed using a Visual Basic2010 program written for this purpose.

\section{The developed Simulation software for the research}

In this paper, the author introduces a VB.net 2010 computer program. The suggested algorithm is applied to a vb.net not a mat lab code (although it is much difficult) to reduce computation loading on the processor. Reducing processing time make it close to industry point of view. Also it is easy to create embedded system (i.e. HIL for a Point scan sensor data processor) [16].The program Graphical user interface GUI is shown in Fig. 1.

\section{The developed algorithm}

The used mathematical model for the unmanned surface vehicle is the so called Nomoto Ship model by Son, K.H. and Nomoto [15]. The algorithm of calculation was created by Guidance Navigation and Control Mat lab toolbox by Tristan Pérez and Trygve Lauvdal [17]. The ship data used is the Mariner Class Vessel data. The author has created a vb.net 2010 program to obtain vehicle model output data in addition to the create point scan sensor simulation code.

The Simulation assumptions are:

1- Each object has a fixed height $\mathrm{H}$ that could change from one object to another.

2- The object shape is a 3D extrusion of a 2D polygon of $\boldsymbol{n}$ number of lines that could change from one object to another.

\subsection{Sensor Simulation Mathematical Model Assumption}

Before proceeding with mathematical modeling two terms must be well defined the first term is the object(s) corners lines, which are the group of lines forming the object shape for the case of rectangle, there are four points and hence four corner lines. These lines are extruded to form a three-dimensional object in this case a parallelepiped.

Fig. 2 shows a wedge-shaped target, (a) two-dimensional sketch. (b) Threedimensional sketch.

The Second term is the scanning line, which is the line beginning at current sensor carrying vehicle position and ends at sensor maximum range at current scan line angle.

The proposed simulation model in this paper was inspired by mathematical model developed by H. Arnaoot [18]. 


\subsection{Mathematical Model Algorithm}

The algorithm could be summarized as follows:

1- Calculate the sensor-carrying vehicle and other object position and orientation in space using the appropriate mathematical model to obtain sensor X, Y, Z-axis position and orientation (in this work Nomoto for surface vehicle and velocity projection for indoor robot).

2- Calculate Scan Line start co-ordinates in X, Y, Z-axis starting from sensor carrying vehicle position and orientation and current sensor scan angle using the following two formulas.

3- Calculate Scan Line end co-ordinates using the spherical co-ordinates, and transfer it back to Cartesian co-ordinates see Error! Reference source not found. Fig. 3.

4- Calculate the co-ordinates of the points forming the simulated object(s). These point co-ordinates change in two cases the first is when the object(s) position change(s); the second is when the object(s) orientation change(s).

5- Calculate the rotation matrix for each object according to it's calculated (pitch, roll, yXaw) angles using the following formulas.

$$
\begin{aligned}
& R_{x}(\theta)=\left[\begin{array}{ccc}
1 & 0 & 0 \\
0 & \cos \theta & -\sin \theta \\
0 & \sin \theta & \cos \theta
\end{array}\right] \\
& R_{y}(\theta)=\left[\begin{array}{ccc}
\cos \theta & 0 & \sin \theta \\
0 & 1 & 0 \\
-\sin \theta & 0 & \cos \theta
\end{array}\right] \\
& R_{z}(\theta)=\left[\begin{array}{ccc}
\cos \theta & -\sin \theta & 0 \\
\sin \theta & \cos \theta & 0 \\
0 & 0 & 1
\end{array}\right]
\end{aligned}
$$

6- Calculate new co-ordinates of points forming the simulated object(s) using the calculated rotation matrix.

7- Calculate intersection point(s) $\mathrm{P}(\mathrm{x}, \mathrm{y})$ co-ordinates between every obstacle corner plane and scan line using algorithm in [19].

8- Moller's algorithm generalizes and optimizes Badouel's. It is based on the transformation of the triangle and the ray so that the triangle is translated to the origin of the coordinate system and is scaled in order to obtain a unit triangle on yz-plane with the ray aligned with the x-axis[20], Fig. 4Error! Reference source not found.

9- By using, the barycentric coordinates of a point with regard to a triangle

$$
S(u, v)=(1-u-v) \cdot v 1+u \cdot v 1+v \cdot v 1
$$

10 - The point is defined as belonging to the triangle if it satisfies $u \geq 0, v \geq 0$ and $u+v \leq 1$. If the ray equation is equaled to the point equation, the intersection point is calculated as follows:

$$
Q 1+t \cdot(Q 2-Q 1)=(1-u-v) \cdot V 1+u \cdot V 2+v \cdot V 3
$$


11-When this equation is solved, the following result is obtained

$$
\left[\begin{array}{c}
t \\
u \\
v
\end{array}\right]=\frac{1}{\left(D \times E_{2}\right) \cdot E_{1}} \cdot\left[\begin{array}{l}
\left(T \times E_{1}\right) \cdot E_{2} \\
\left(D \times E_{2}\right) \cdot T \\
\left(T \times E_{1}\right) \cdot D
\end{array}\right]
$$

12-Remove fake scan matrix results using methods proposed by [18].

13-Applying previous equations with the scan line and each obstacle corner plane yields a set of points.

14-For each detected point, calculate the distance between intersection point and sensor carrying vehicle current position.

15-The resulting scan time, scan angle and range are the sensor output data.

\section{Algorithm Verification}

\subsection{Algorithm Verification Overview}

In general, the evaluation of a simulation technique is done by comparing the simulated data with the real data for the same situation. In the current case it very difficult to do so. Because in sea trials the environmental conditions (wind, current, tide) cannot be accurately predicted and-or simulated. In addition a significant part of technique evaluation will involve environmental conditions prediction and-or simulation accuracy, which is not the main scope of the current work.

In the current search the verification was done by logging test vessel navigational data (roll, pitch, yaw, heave, sway, surge, ship position and ship speed) and sensor data. The logged navigational data is fed to sensor simulation algorithm and the run a comparison between the actual collected and simulated sensor data. By using this technique the environmental conditions effect is nearly eliminated. This technique verifies the sensor simulation part of the algorithm isolated from the ship simulation part to focus on the current search scope.

\subsection{Equipment Used In Algorithm Verification Technique Overview}

The ship used is an eleven meters long with 3-ton displacement, propulsion system is two 200 horse power outboard engines which makes a total of 400 horse power, See Fig. 5. The boat has achieved a maximum speed of 35 knots in calm sea state.

There are three main sensors used in the verification process the point scan sensor which is selected as Sick LMS221, the differential Global positioning system device DGPS which is selected as differential GPS Trimble R7 and GPS Receiver, the gyro compass which is selected as gyrotrac and the displacement - rotation measuring device (to determine roll and pitch)

\subsubsection{The laser measurement system (Sick LMS221)}

The laser measurement system (Sick LMS221)[21] is mounted on top of the boat super structures on the middle. The distance value per individual impulse (spot) is evaluated. This means that a distance value is provided every $0.25^{\circ}, 0.5^{\circ}$ or $1^{\circ}$, depending on the selected angular resolution of the sensor. In the current work the simulation as well as sea trials was 
carried out using $1^{\circ}$ resolution. The sensor data is collected through serial port (RS 232) interface to main computer.

\subsubsection{Gyro compass}

The Gyro compass is used to estimate the absolute ship heading (yaw) and hence absolute sensor heading, a Gyrotrac is selected.

\subsubsection{Differential GPS}

A differential GPS Trimble R7 GPS and Receiver is used to estimate the absolute ship position (easting and northing), data is collecting through a serial port with Baud rates 9600 bps. The Output format is NMEA-0183. The estimated error is about 0.5 to 1.0 meter.

\subsubsection{IMU unit}

An IMU is used to estimate the ship orientation in space roll, pitch and yaw. The yaw data is obtained from the gyro compass.

\section{RESULTS}

\subsection{Results Preface}

As previously illustrated, a number of sea trials were carried out. During the sea trails, test vessel navigational data as well as sick 218 sensor data was logged. The logged navigational data was fed to the suggested sensor simulation algorithm.

\subsection{Test Boat Logged Data}

The figures Fig. 6 to Fig. 8 are part of a field sea trial logged data during turning in a full circle.

\subsection{Comment on the result}

As seen when comparing Fig. 9 with Fig. 10, the real scan matrix data is relatively uniform, while the simulated scan matrix data is not as uniform as the real.

The lack of uniformity is believed to be due to data sensors error. To verify this theory a random error was injected into to the simulated data. The results showed lack of uniformity that increase with injected error margin increase.

\section{Conclusion}

1. Simulation is a key element in the design of autonomous vehicles in general and in USV in specific.

2. The vehicle motion has great impact on the quality of on board sensor data quality.

3. The ship pith and roll amplitudes motion along with sensor mounting height with respect to sea level are the major factor that determines the fake point's number.

4. Environment identification sensor are sensitive to position in space sensor specially IMU sensor.

5. The synchronization between position sensors is very important specially when the Environment identification sensor scan speed is comparable to:

a- Change of ship position or orientation in space 
b- Rate of ship position or orientation in space sensor data output

\section{Future Work}

1- Create an algorithm to analyze sensor error and uncertainty and their effect on range error and test it.

2- Create hardware in the loop to test ship control techniques.

\section{Figures}
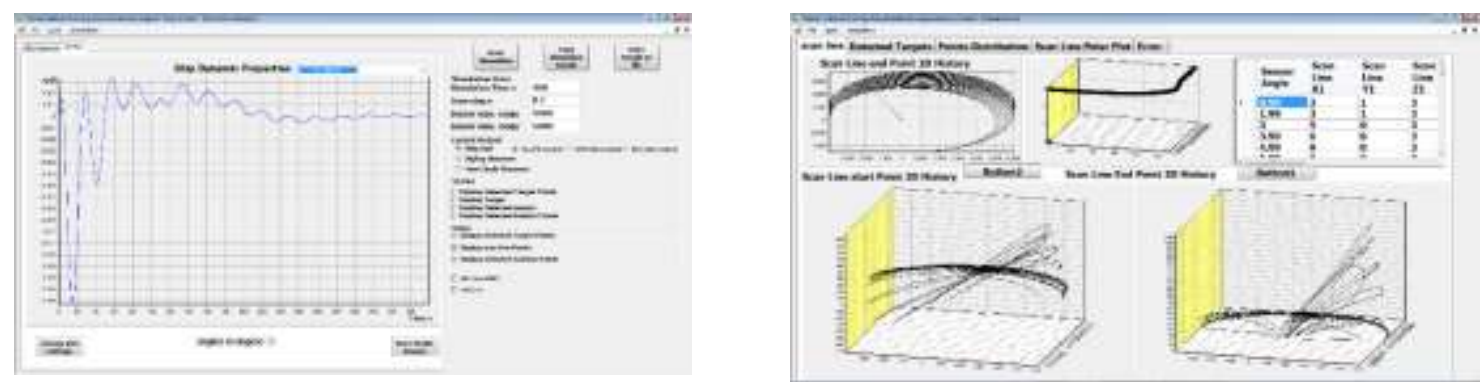

Fig. 1: Graphical User Interface GUI of the suggested VB.net 2010 computer program (a) Ship dynamic Characteristics change versus time,(b) Sensor scan Characteristics.

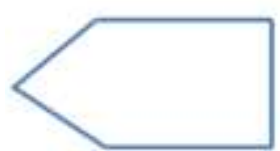

Figure $A$

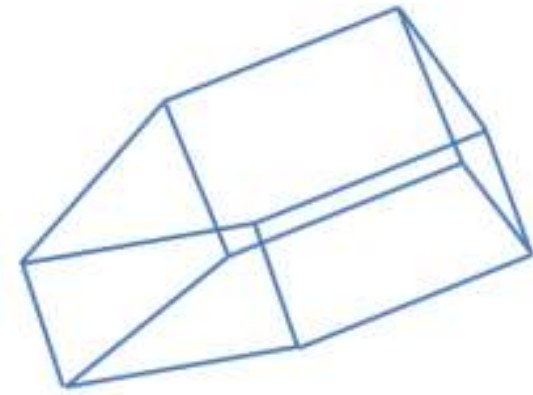

Figure o

Fig. 2: A wedge-shaped target (a) 2D sketch, (b) 3D sketch.

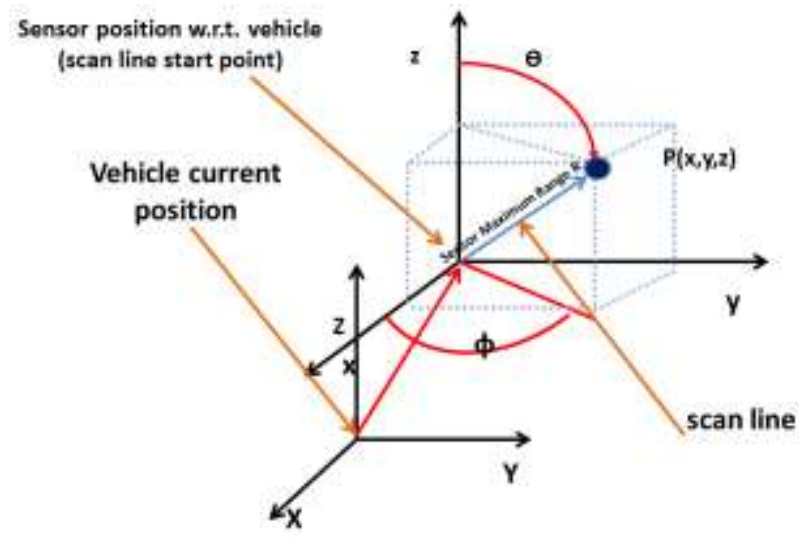

Fig. 3: Relation between scan line start, ends, and vehicle position. 

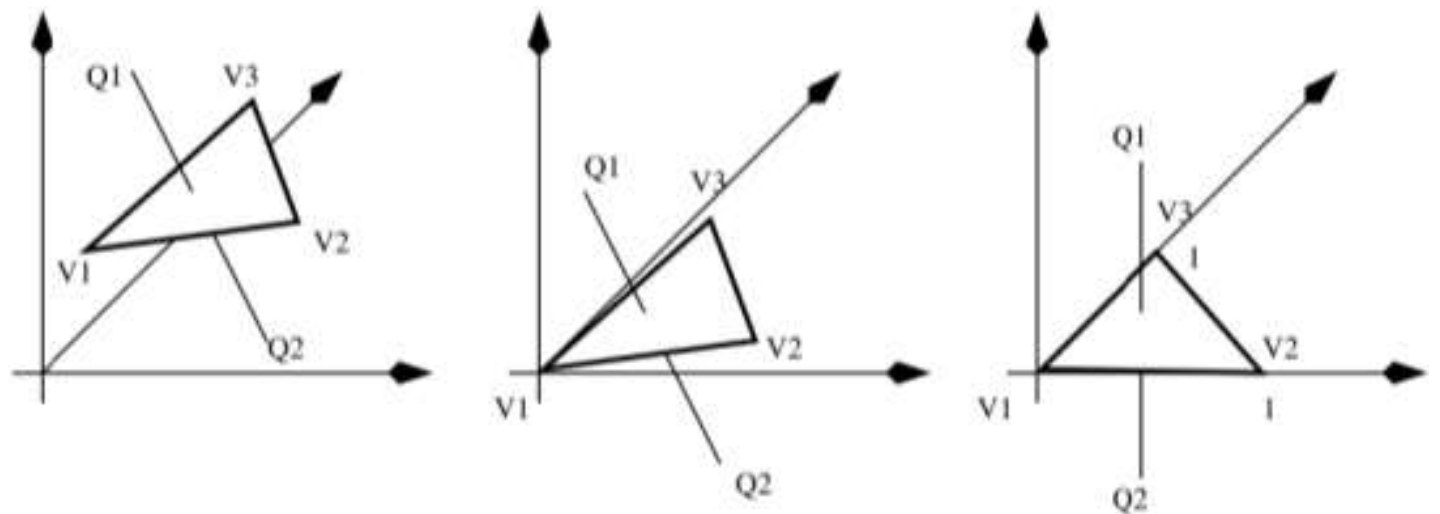

Fig. 4: Illustration of Moller's algorithm

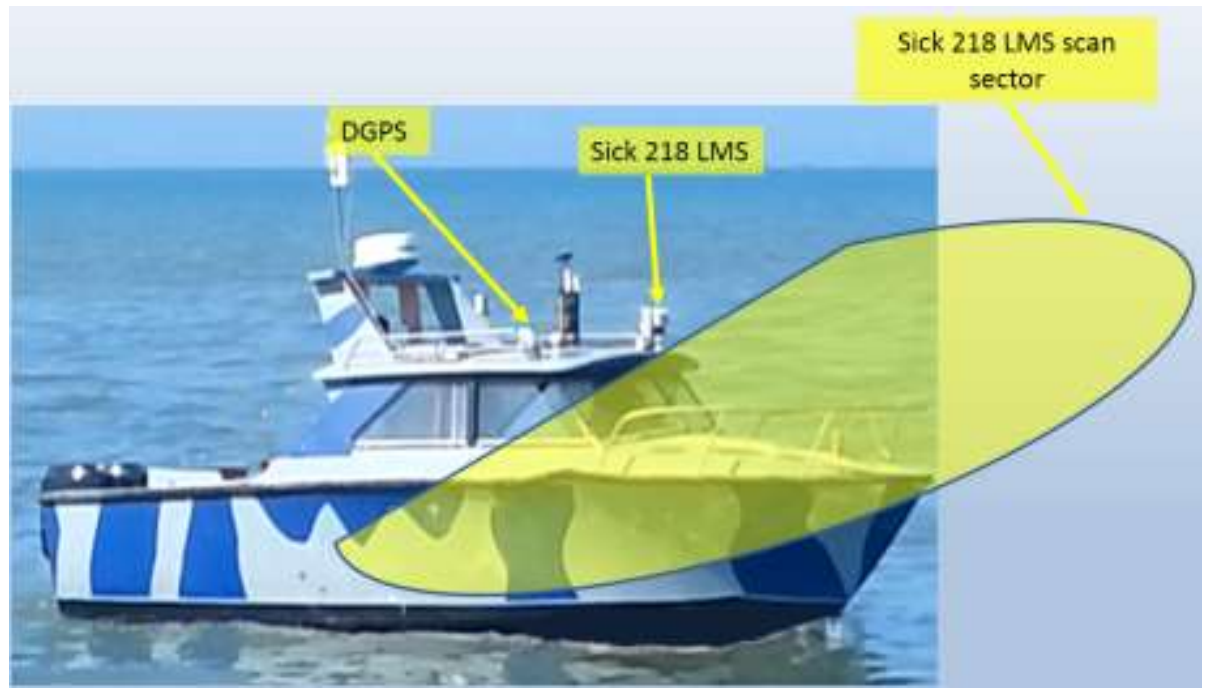

Fig. 5: the yacht used for sea trials.

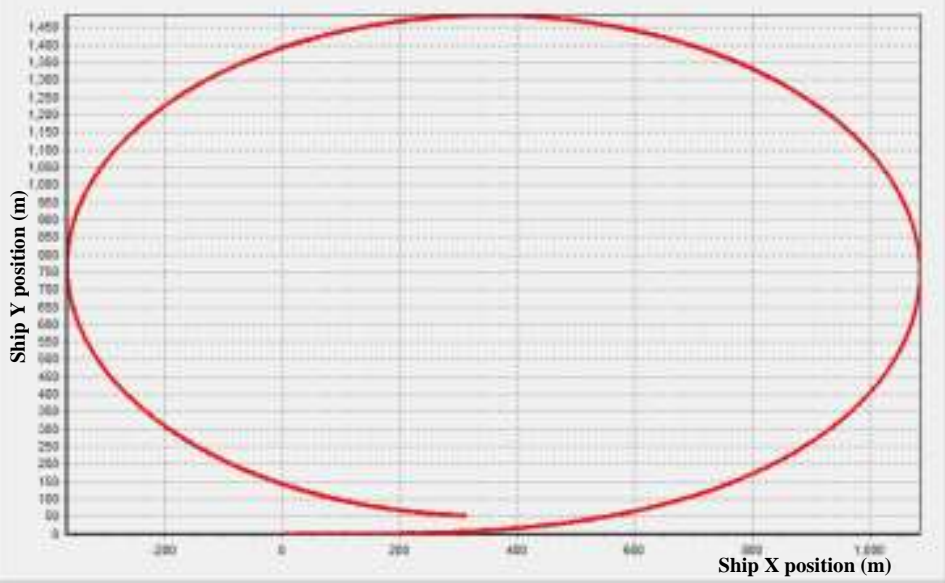

Fig. 6: Field sea trial logged data during turning in a full circle (ship Postion) 


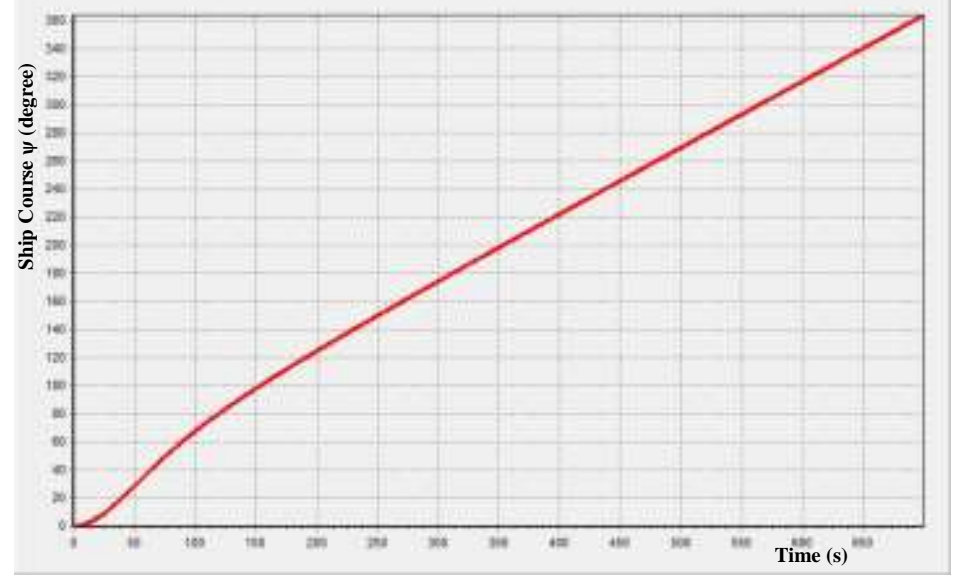

Fig. 7: Field sea trial logged data during turning in a full circle (ship course)

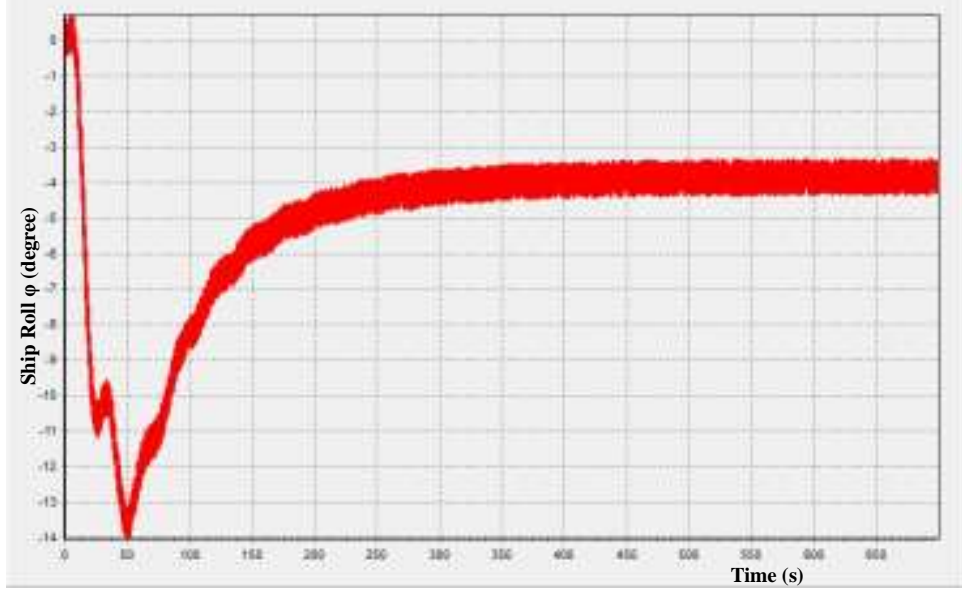

Fig. 8: Field sea trial logged data during turning in a full circle (ship roll angle)

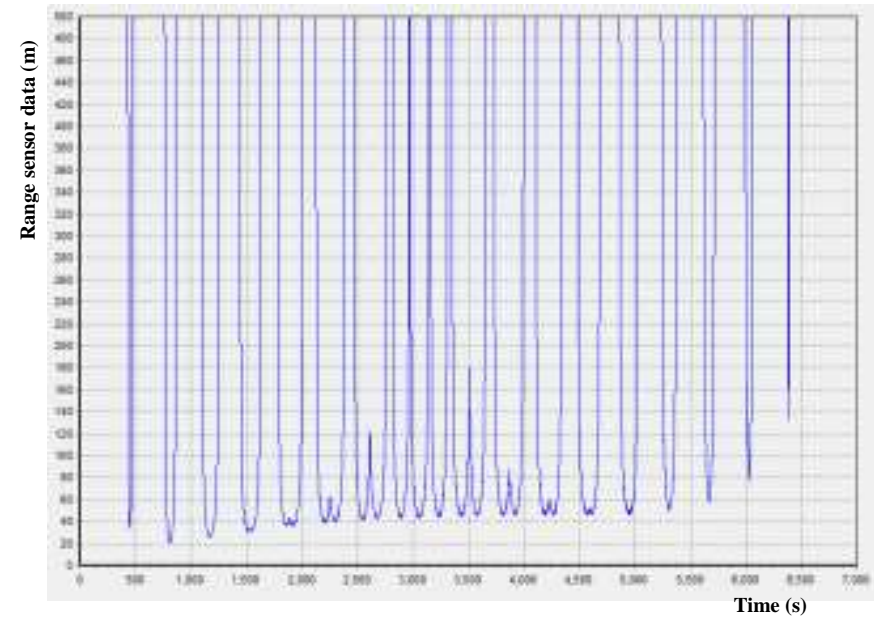

Fig. 9: Real range sensor data versus time based on collected Field sea trial logged data during turning in a full circle 


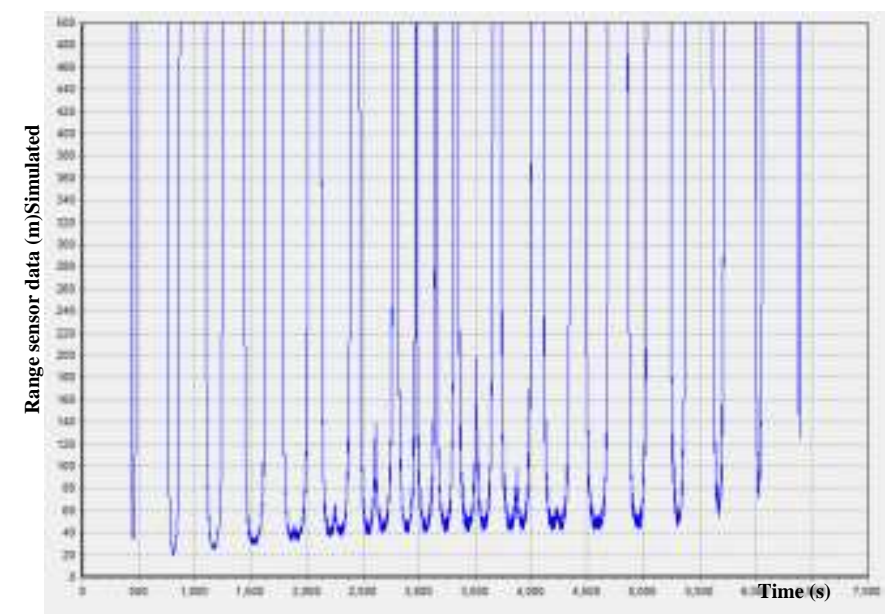

Fig. 10: Simulated range sensor data versus time based on collected Field sea trial logged data during turning in a full circle

\section{Reference}

[1] SungTae Moon, W.E., Hyunchul Gong, Development of Large-Scale 3D Map Generation System for Indoor Autonomous Navigation Flight - work in progress "APISAT2014", 2014 Asia-Pacific International Symposium on Aerospace Technology, elsevier, 2015. 99(): p. 5.

[2] Russell B. Wynn, V.A.I.H., Timothy P. Le Bas Autonomous Underwater Vehicles (AUVs): Their past, present and future contributions to the advancement of marine geoscience, elsevier. elsevier, 2014. Marine Geology 352: p. 18.

[3] Hans-Christoph BURMEISTER, W.B., Ørnulf Jan RØDSETH, Thomas PORATHE, Autonomous Unmanned Merchant Vessel and its Contribution towards the e-Navigation Implementation: The MUNIN Perspective. International Journal of e-Navigation and Maritime Economy, elsevier, 2014. 1: p. 13.

[4] Glenn A. Osga, M.R.M., Human-computer interface studies for semi-autonomous unmanned surface vessels. Procedia Manufacturing , elsevier, 2015. 3(-): p. 8.

[5] Anish Pandey, D.R.P., MATLAB Simulation for Mobile Robot Navigation with Hurdles in Cluttered Environment Using Minimum Rule Based Fuzzy Logic Controller. 2nd International Conference on Innovations in Automation and Mechatronics Engineering,ICIAME 2014 , elsevier, 2014. 14(-): p. 7.

[6] Deschaud, J.E., Prasser , D., Dias, M.F., Browning, B., Rander,P. , Automatic Data Driven Vegetation Modeling for LIDAR Simulation. U.S. Army Engineer Research and Development Center, (ERDC) under cooperative agreement "Fundamental Challenges in World and Sensor Modeling for UGV Simulation, 2012. (Number W912HZ-09-2-0023): p. 7.

[7] standards, N.c.f.g.i., Light Detection and Ranging LIDAR Sensor Model Supporting Precise Geopositioning. 2013, USA: National center for geospatial intelligence standards. 5.

[8] Peinecke, N., Lueken, T., Korn, B.R., , LIDAR simulation using graphics hardware acceleration. Digital Avionics Systems Conference, 2008. DASC 2008. IEEE/AIAA 27th, 2008. 4: p. 8. 
[9.Gryaznov , N.a.L., A., , Computer Vision for Mobile On-Ground Robotics. 25th DAAAM International Symposium on Intelligent Manufacturing and Automation, 2014: p. 5.

[10] Dula Nad, N.M., Filip Mandi, Navigation, guidance and control of an overactuated marine surface vehicle. Elsevier, 2015. 40(2015): p. 172-181

[11 Claudio Urrea, J.K., Design, simulation and comparison of controllers for a redundant robot. Case Studies in Mechanical Systems and Signal Processing, elsevier, 2016. 3(3): p. 13.

[12] James Underwood, A.H., Dr. Steve Scheding, Calibration of Range Sensor Pose on Mobile Platforms. IEEE/RSJ International Conference on Intelligent Robots and Systems, 2006(2007): p. 6.

[13] A. Nuchter, K.L., J. Hertzberg, and H. Surmann, 6D SLAM - Mapping Outdoor Environments. Journal of Field Robotics, 2007. 24(8-9): p. 699-722.

[14] F. Pomerleau, F.C., R. Siegwart, and S. Magnenat, Comparing ICP Variants on RealWorld Data Sets Open-source library and experimental protocol. Autonomous Robots, 2013. 34(3): p. 133-148.

[15] Son , K.H.a.N., On the Coupled Motion of Steering and Rolling of a High Speed Container Ship, Naval Architect of Ocean Engineering. J.S.N.A., 1982. 150: p. 10.

[16] Khalid bin Hasnan, L.B.S.a.T.H. and A Hardware-In-the-Loop Simulation and Test for Unmanned Ground Vehicle on Indoor Environment 2012 International Workshop on Information and Electronics Engineering (IWIEE) 2012. 29: p. 5.

[17] Tristan Perez, T.L., guidance, navigation and control Matlab toolbox, in guidance, navigation and control Matlab toolbox, 2008.

[18] Gamal Zaghloul, H.A., Simulation of a Laser Measurement System LMS Sensor Mounted on a Moving Vehicle Detecting Moving Obstacles. Proceedings of the 16th International Conference on Aerospace Sciences and Aviation Technology, 2015. 1: p. 11.

[19] Ericson, C., Real-Time Collision Detection. The Morgan Kaufmann Series in Interactive 3D Technology, ed. M.S. David H. Eberly, Inc. Vol. 1. 2005, United States of America: Morgan Kaufmann Publishers is an imprint of Elsevier. 633.

[20] Juan J. Jiménez, R.J.S., Francisco R. Feito, A robust segment/triangle intersection algorithm for interference tests. Efficiency study. Elsevier, Computational Geometry 2010. 43: p. 474-492.

[21] LMS200/211/221/291 Laser Measurement Systems TECHNICAL DESCRIPTION, S.A. Waldkirch, Editor. 2006. 